\title{
Coupling Browse and Search in Highly Interactive User Interfaces: A Study of the Relation Browser++
}

\author{
Junliang Zhang \\ Interaction Design Lab \\ School of Information and Library Science \\ University of North Carolina, Chapel Hill \\ 919-962-8274 \\ junliang@email.unc.edu
}

\author{
Gary Marchionini \\ Interaction Design Lab \\ School of Information and Library Science \\ University of North Carolina, Chapel Hill \\ 919-966-3611 \\ march@ils.unc.edu
}

\begin{abstract}
A novel interface called the Relation Browser++ $(\mathrm{RB}++)$ for searching and browsing large information collections was designed and investigated. RB++ provides visualized category overviews of an information space and allows dynamic filtering and exploration of the result set by tightly coupling the browsing and searching functions. A user study was conducted to compare the effectiveness, efficiency and user satisfaction of completing various types of searching and browsing using the RB++ interface and a traditional form-fillin interface for a video library. An exploration set of tasks was also included to examine the effectiveness of and user satisfaction with the RB++ when applied to a large federal statistics website. The comparison study strongly supported that $\mathrm{RB}++$ was more effective and efficient for completing designed tasks, especially those of data exploration, and it gained higher satisfaction from users. The exploration study showed that the interface not only facilitated users to have better understanding of the web based information collection but also was effective and efficient to find needed information from it.
\end{abstract}

\section{Categories and Subject Descriptors}

H.5.2 [Information Interfaces and presentation (e.g. HCI)]: User Interface - interaction style, graphical user interfaces (GUI)

H.3.3 [Information Storage and Retrieval]: Information Search and Retrieval - query formulation, search process, selection process

H.3.7 [Information Storage and Retrieval]: Digital Libraries User issues, Systems issues

\section{General Terms}

Design, Human Factors, Experimentation, Performance

\section{Keywords}

Interface design, search, browse, category overview, visualization, dynamic query, interactive system

\section{INTRODUCTION}

The size and breadth of digital libraries makes it difficult for people to quickly grasp what content is and is not available. Consequently, people usually need an overview of the digital library to help them decide if it is worthwhile to look further. As they do look further, it is helpful for their searching and browsing to get an idea of what is in the collection and how many items are available. We believe that digital library users will be well-served by highly interactive user interfaces that support alternative views of the library partitions/collections/results sets. This paper describes a user interface that aims to provide agile control over various partitions and reports results from a user study comparing this interface to a typical library search interface.

Faceted category structure is one way to help people understand the composition of an information collection. A Faceted approach provides different ways to slice and dice the information space, which allows the users to look at the information space from different perspectives. Allowing people to explore the relationships among different facets may further deepen their understanding and support new insights. The relational browser $(\mathrm{RB})$ is an interface which provides an overview of the collection by displaying different categories and enables people to explore the relationships among these categories [10]. The different facet values also serve as selectable objects that may be employed as query widgets for a search so that the entire space can quickly be partitioned with simple mouse moves and with consequent immediate display of the resulting partition in the results panel. Figure 1 shows an application of an early version of the relational browser in the domain of the U.S. federal statistics websites. The web pages in the site were sliced into four different facets: by topic, data type, region, and date. The numbers beside the bars indicate the number of web pages associated with the attributes. By mousing over any of the topics, distribution of the specific topics in other facets are visualized as graphic bars. 


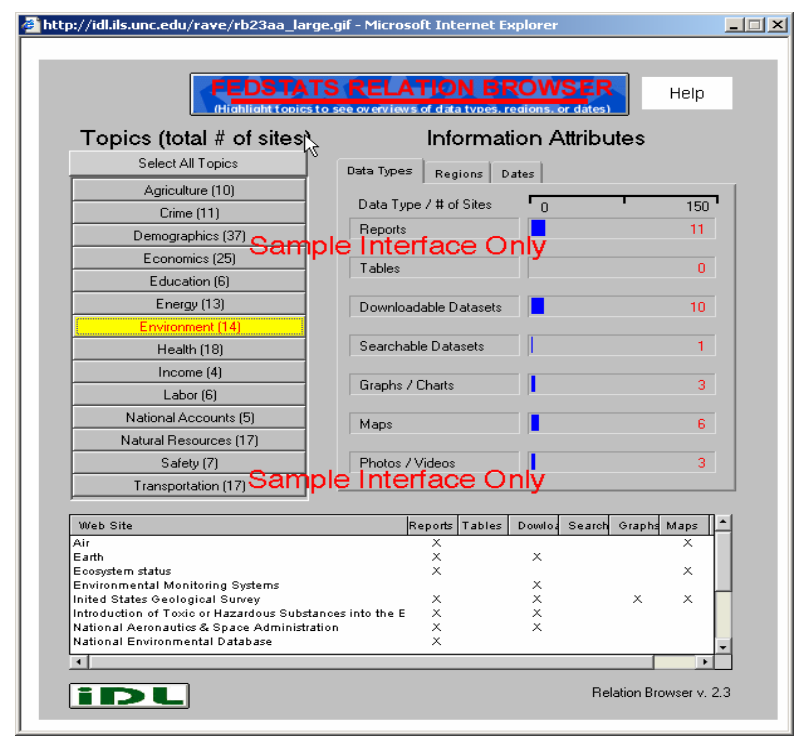

Figure 1. Relational Browser (RB)

Recently, we updated this early version of the RB [20]. The new version is called $\mathrm{RB}++$, which improves the $\mathrm{RB}$ significantly in several ways (see Figure 2). First, RB++ displays multiple facets (categories) visually and on the same screen rather than only two facets with tab options to others. The multiple facets provide an overview of the information space. The facet values are visually represented by graphic bars with different lengths, which indicate the number of items associated with them. Second, $\mathrm{RB}++$ allows more flexibility to explore relationships. One of the features of $\mathrm{RB}++$ is that you can restrict the information items (partition the information space) by mousing over any bars and other bars are proportionally highlighted to show the conditional distribution. Note that you can only utilize the bars under one 'main' facet to get the conditional distribution in previous $\mathrm{RB}$ versions. Third, the $\mathrm{RB}++$ added a dynamic filtering function for the result set (see Figure 3). Once the search results are displayed in the table, further filtering can be done by typing in keywords (string patterns) in the boxes located immediately above the result fields. The filtering is dynamic, which means that with each character typed in or removed from the boxes, RB++ matches the string patterns in the boxes with the corresponding field of the results. Only the matched results are then displayed immediately in the results panel and the matched string in the results is highlighted. This dynamic feature gives users instant and constant feedback about the filtered results and how many items they will get with different keywords, which allows the users try out different filtering keywords very easily and efficiently. Fourth, the RB++ provides an overview of the results set and tightly couples the overview and results set panels. The overview panel is dynamically updated to give users a contextualized overview of updated result set. These new features give users more power to understand and explore the information collection and give them a flexible and rapid way to find the information they want. A linguistic model of BNF grammar to model the user interaction with the interface is provided in section 2.3 to help reveal the dynamic nature of the $\mathrm{RB}++$. We argue that the $\mathrm{RB}++$ interface will bring users added values beyond simple searching and browsing by in fact combining these search strategies seamlessly. In the next section, the methodology of a user study is described. The results of the user study are then presented, followed by a discussion of the results. Related work is then presented and limitations of the interface and future work are discussed.

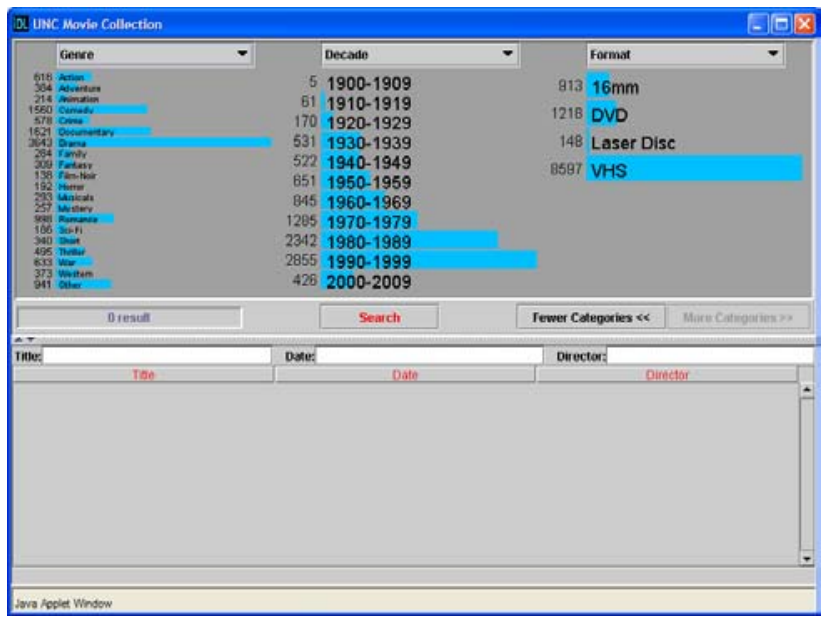

Figure 2. Initial display of RB++ with visualized category overview on the top

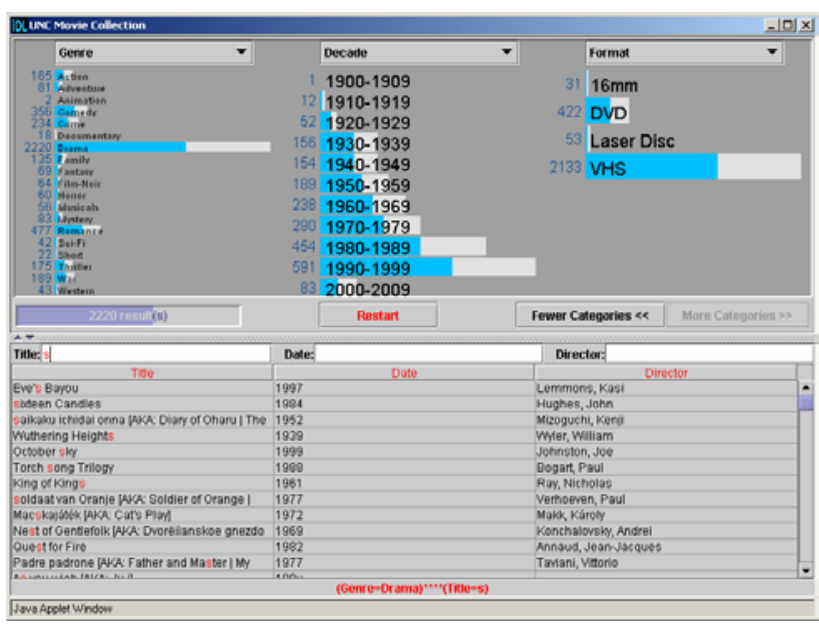

Figure 3. RB++ with dynamic filtering of the results (note the changes in the overview and updated results)

\section{METHODOLOGY}

The purpose of the user study was two-fold: first, we wanted to compare the effectiveness, efficiency and user satisfaction associated with completing certain tasks using $\mathrm{RB}++$ against that obtained by the traditional form-fillin search interface (baseline interface). Second, we wanted to explore if the RB++ interface would lead to new interaction patterns with the interface and what these new interaction patterns might be?

Seventeen undergraduate and graduate students were recruited from the UNC-Chapel Hill campus for this study. They came from various schools and departments such as the School of Information and Library Science, and the Psychology, English, and Mathematics departments. Among all the participants, there were 10 females and 7 males. The ages of the participants ranged from 19 to 44 , but 15 of them were in their 20 s. They were all familiar with www browsers. The participants were 
given $\$ 15$ for their participation. The data from the first two participants was used as a pilot test; based on which the experimental protocol and instruments were revised. The data for the other 15 participants was used for the data analysis. The test protocol was the same for all the participants.

\subsection{Phase One: Compare $\mathrm{RB}++$ to Baseline for Films}

The study was composed of two phases. The first phase was a comparison study. Participants used both the $\mathrm{RB}++$ interface and the baseline interface. The order of using these interfaces was counter balanced. The domain of the information items in both interfaces was the video collection in the UNC-CH library (http://www.lib.unc.edu/house/mrc/index.html?page=filmograph y). The library online video search interface (Filmfinder) was used as our baseline interface. FilmFinder is a fairly typical www form-fillin search interface (see Figure 4 and Figure 5), where users can specify queries within fields such as title, release year, director, description, genre, origin, and format.

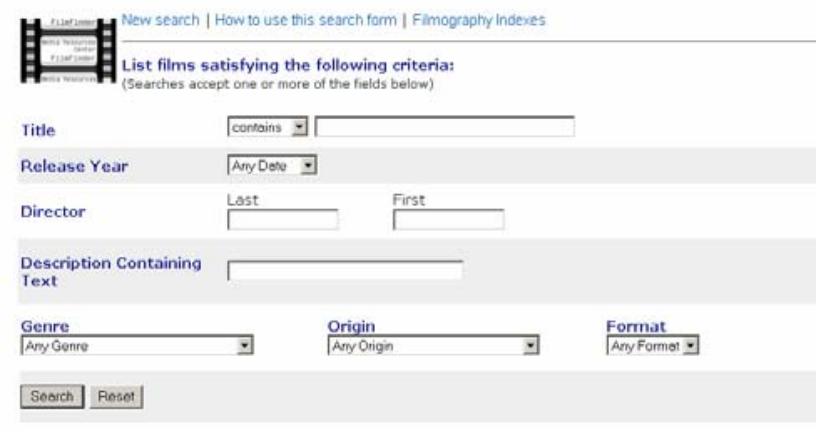

Figure 4. Filmfinder with Form-fillin Interface

Media Resources Center Filmography Search MRC Home | Filmfinder

Records 1 - 50 of 99 film(s) that match your query in the Media Resources Center Collection. Previous: none Current: 1 - 50

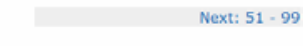

order by

Title

1:: 40 Days and 40 Nights

- Forty Days and Forty Nights

2: About a Boy
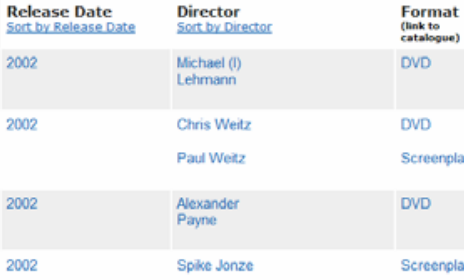

DVD

Figure 5. Results Page of Filmfinder

The protocol for the first phase was as follows: First, a demographic pre-test questionnaire was filled out. Second, the participant was trained for the first interface assigned to in their condition. The training consisted of: an introduction to the features of the interface, a demo of each type of task with the interface, and participant practice using the interface until s/he was comfortable with it. Third, the participant used the interface to complete 10 search tasks. Tasks were assigned to participants one by one by handing them pieces of paper for each task. A timer was used to count time used to complete each task except for task 10 (see description of task 10 below). After each task, a short satisfaction questionnaire was completed by the participant. Fourth, a usability questionnaire was filled out after participant finished using the first interface. Next, the participant was trained for the second interface and the same procedures were used to complete 10 more search tasks. Finally, an open questionnaire about perceived differences and preferences for the two interfaces was completed.

All participants were run individually in sessions ranging from 60-90 minutes. All sessions were video taped.

The tasks were classified into three different types: 1. Simple look up task. Tasks 1 to 3 in each task set are of this type. For example, "Check if the movie titled "The Matrix" is in the library movie collection." 2. Data exploration and analysis tasks. Tasks 4 to 9 in each task set are of this type. This kind of task requires users to understand and make sense of the information collection, which could be a starting point for them to further their searching or browsing. Two examples of this type are: "In which decade did "Steven Spielberg" direct the most movies?"; and "How many movie titles does the library hold that were released in the year 2000?" 3. Task 10 was a free exploration task, which asked participants to find five favorite videos without counting the time spent on it. The tasks assigned for the two interfaces were different but comparable. For example, the comparable tasks for two interfaces simply substituted different video titles or directors.

\subsection{Phase Two: Explore RB++ for Energy Website}

The second phase was an exploratory study on the RB++, which was applied to a different domain: the website for the Energy Information Administration (EIA), which contains roughly 10,000 web pages. The web pages were classified into four different facets: fuel type (with the facet values: alternatives, coal, electricity, natural gas, nuclear, petroleum, and renewable); geography (state level, regional level, national level, and international level); sector (commercial, electric utility, industrial, and residential); and process (delivery, import/export, price/cost, production, resources/reserves, and usage). All the facets were displayed on the overview panel (see Figure 6). The results panel displayed the title, page size, and description of the web pages.

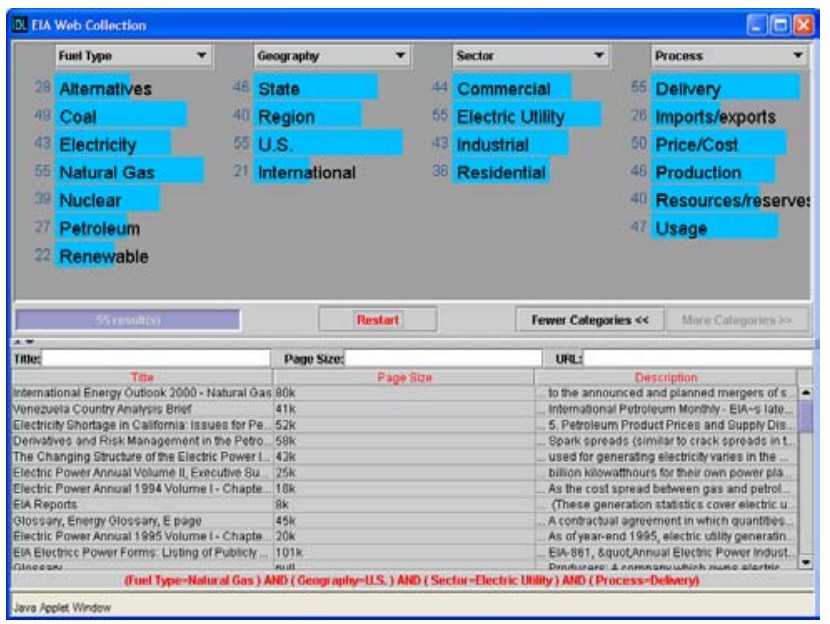

Figure 6. RB++ interface applied to EIA website 
The protocol of the second phase was composed of: First, RB++ with EIA application was introduced to the participant. Second, the participant practiced the interface until s/he was comfortable with it. Third, the participant used the interface to complete four tasks ${ }^{1}$. The process was recorded and a short satisfaction questionnaire was filled out after finishing each task. Fourth, an open-ended questionnaire was completed after finishing all the tasks. Lastly, the participant was briefly interviewed.

Data collected included both quantitative and qualitative data from the two phases of the study. Data we collected for the first phase included performance data (time spent finishing tasks), error rates of tasks, ratings on the satisfaction questionnaire after finishing each task, ratings on the usability questionnaire after finishing each interface, and comments on the open questionnaire about perceived differences and preferences for the two interfaces. Data collected for the second phase included ratings on the satisfaction questionnaire after finishing each task, comments on the post-session questionnaire and the verbal comments made in the interview.

\subsection{Modeling User Interaction}

In order to help us form hypotheses and analyze and make sense of the experimental data, we employed a linguistic model, called BNF grammar, to model the user's interaction with the interface. BNF grammar was originally used by Reisner to describe the dialog grammar of an interactive graphics system [13], where the user's interaction with a system was seen as an action language and BNF grammar was used to formally describe the language. The BNF grammar consists of a set of rules which define higher level user behaviors in terms of lower level ones. Each rule is composed of terminals, non-terminals, and a set of symbols. Terminals usually represent the lowest level of user behavior, such as pressing a key or clicking a mouse button and can not be further defined. Non-terminals represent a high level abstraction and can be defined in terms of other non-terminals and terminals. Terminals are written with upper case letters and non-terminals are written with lower case letters. The "::=" symbol is read as " is defined as". The "+", "|" and "-. symbols are used at the right hand side of rules to connect, respectively, sequence of user behavior, set of options, and concurrent user behaviors. With the BNF grammar, we can describe the user's interaction with the $\mathrm{RB}++$ as follows:

A1 information seeking ::= explore collection(A3) | (formulate query(A2) + CLICK SEARCH BUTTON + navigate results(A5))

A2 formulate query ::= (explore collection(A3) + form query(A4)) | form query(A4)

A3 explore collection ::= (CLICK VISUAL BAR-OBSERVE VISUAL BAR + explore collection(A3)) | (MOUSE OVER

\footnotetext{
${ }^{1}$ Tasks for the second phase study:

1. I want to learn the current status of Chinese nuclear energy.

2. Find the most recent weekly data on the petroleum price in the USA.

3 . Find the statistical data on coal production across different states in the year 2001 .

4. What kinds of information can I and can not I find from the website?
}

VISUAL BAR-OBSERVE VISUAL BAR + explore collection(A3))

A4 form query ::=(CLICK VISUAL BAR + form query(A4)) (TYPE IN KEYWORD + form query(A4))

A5 navigate results ::= (browse results(A6) + navigate results(A5)) | (CLICK RESTART BUTTON + information seeking(A1))

A6 browse results ::= (show results(A7)-OBSERVE RESULTS + browse results(A6)) | (CLICK RESULT ITEM + browse results(A6)) | (CLICK SORTING BUTTON + browse results(A6)) $\mid$ (explore results(A8) + browse results(A6))

A7 show results ::= CLICK SIDEBAR

A8 explore results ::= (observe system state(A9) + explore results(A8)) $\mid$ (filter results(A10) + explore results(A8))

A9 observe system state ::= (OBSERVE VISUAL BAR + observe system state(A9)) | (OBSERVE NUMBER + observe system state (A9))

A10 filter results ::= CLICK VISUAL BAR | MOUSE OVER VISUAL BAR | TYPE IN KEYWORD

The interaction with baseline interface can be described as:

B1 information seeking ::= formulate query(B2) + CLICK SEARCH BUTTON + navigate results(B4)

B2 formulate query ::= (TYPE IN KEYWORD + formulate query(B2)) $\mid($ select item(B3) + formulate query(B2))

B3 select item ::= CLICK PULL DOWN MENU + CLICK ITEM

B4 navigate results ::= (browse results(B5) + navigate results(B4)) | (CLICK NEW SEARCH LINK + information seeking(B1))

B5 browse results ::= (show results(B6)-OBSERVE RESULTS + browse results(B5)) $\mid$ (show results(B6)-COUNT RESULTS + browse results(B5)) $\mid$ (CLICK ITEM + browse results(B5)) $\mid$ (CLICK SORTING LINK + browse results(B5))

B6 show results ::= CLICK SIDEBAR | (CLICK SIDEBAR + CLICK NEXT PAGE LINK)

The number of rules and options within rules reflects the interactive nature and number of alternative choices provided by these two interfaces. Note that we used the terminals such as CLICK SEARCH BUTTON and CLICK VISUAL BAR which strictly speaking are not the lowest level of user behaviors, however, using higher level abstraction as terminals is suitable for interactive display-based systems [4] and ensures later data analysis. Many rules are defined recursively and consist of several options, which essentially reflect the interactivity of graphical user interface (GUI). For example, a fairly interactive user behavior in RB++, "browse results (A6)", consists of either 'OBSERVE RESULTS', 'CLICK RESULT ITEM', 'CLICK SORTING BUTTON', explore results, or any combination of the above.

From the BNF definition, we can see that $\mathrm{RB}++$ is a more interactive interface than the baseline since it involves more rules and recursive definitions. However, it is not necessarily a complicated interface, since the rules for $\mathrm{RB}++$ interface are 
largely composed of set of options instead of sequence of user behaviors, which means that many rules are not executed for some types of tasks. Based on the BNF grammars, hypothesize that for the simple search tasks, the $\mathrm{RB}++$ interface will not necessarily be significantly different from the baseline interface, but for the complicated searching and browsing tasks, that require more interaction or collection exploration, the $\mathrm{RB}++$ will be significantly more effective, efficient and gain more user satisfaction than the baseline. For simple look up type tasks, both interfaces involve the sequence of user actions: formulate query, CLICK SEARCH BUTTON, and navigate results (see rule A1 and B1). Navigation of results is simple for this type of task in that it only involves the judgment of zero or non-zero results, which is trivial in both interfaces. Formulation of the query in this case involves typing in keywords and/or selecting the items from the interfaces (see rule A2, A4 and B2, B3). Even though item selection in the baseline interface involves two clicks (see rule B3) which means a slightly longer time to execute than in $\mathrm{RB}++$, which only needs one click on visual bar for item selection (see rule A4), we expected no significant difference on this aspect in our study (for relatively small number of participants). For type 2 tasks that involve data exploration and analysis, interaction with the visual bars of the $\mathrm{RB}++$ interface provides an effective and efficient interaction style. Two typical sequences of user behaviors to complete type 2 tasks are: explore the collection by clicking (or mousing over) and observing the visual bars (see rule A1 and A3), or formulate query and then explore the results by observing the visual bars (see rule A1, A5, A6, A8 and A9). With the traditional interface to finish type 2 tasks, users have to formulate a query and then literally scan and count all the results (see rule B1, B4, B5, and B6), which is time consuming.

We also hypothesized that users would exhibit rich interaction during their navigation of the results with $\mathrm{RB}++$ (see rule $\mathrm{A} 5$ to A10). Actions of typing in keywords and clicking visual bars to filter results (rule A10) would be used frequently and interchangeably by the users to finish complex search tasks, especially when large numbers of results are returned.

\section{RESULTS}

\subsection{Phase One Results}

Table 1 lists the average time (in seconds) across all the participants to finish task 1 to task 9 using the two different interfaces. Notice that we allowed the participants to stop the task if they felt that the task was hard or time-consuming to finish. It turned out that there were five participants who stopped task 5 and eight participants stopped task 6 before completion when they used the FilmFinder. Performance data of these participants were discarded for the unfinished tasks.

Table 1. Performance data (in seconds)

\begin{tabular}{|c|c|c|c|c|c|}
\hline Task & $\mathbf{1}(\mathbf{. 8 7 9 )}$ & $\mathbf{2} \mathbf{( . 5 2 2})$ & $\mathbf{3}(\mathbf{. 0 2 6})$ & $\mathbf{4}(\mathbf{. 0 0 0})$ & $\mathbf{5}(\mathbf{. 0 0 0})$ \\
\hline RB++ & 14.4 & 16.1 & 17.0 & 18.9 & 15.7 \\
\hline FilmFinder & 14.7 & 14.4 & 29.7 & 40.7 & 204.0 \\
\hline Task & $\mathbf{6 ( . 0 0 0 )}$ & $\mathbf{7 ( . 0 0 0 )}$ & $\mathbf{8 ( . 0 0 0 )}$ & $\mathbf{9}(.000)$ & $\mathbf{1 0}$ \\
\hline RB++ & 12.7 & 13.5 & 27.1 & 20.6 & N/A \\
\hline FilmFinder & 328.0 & 87.2 & 101.3 & 112.8 & N/A \\
\hline
\end{tabular}

Paired sample $t$ tests on the performance data were computed and the $p$ values are shown in the parenthesis for each task. We can see that except for the first two tasks (which were type 1 tasks), the performance differences between the two interfaces were all significant at least the $2.6 \%$ level. Clearly, RB++ supported superior performance for type 2 tasks.

We also counted error rates for task 1 to task 9, which are listed in table 2. The error rate was calculated as the number of participants who gave the wrong answer to the task divided by total number of participants. We can see that except for the 8th task, no participants got wrong answers for any of the tasks using the $\mathrm{RB}++$ interface. The error rates of the baseline interface were much higher than that of the $\mathrm{RB}++$ interface, especially for tasks 5,6 , and 7 . Notice that we did not consider those participants who gave up the task 5 or 6 using the Filmfinder, so the actual denominators used for calculating the error rates for these tasks were smaller than the total number of participants.

Table 2. Error rates

\begin{tabular}{|c|c|c|c|c|c|}
\hline Task & $\mathbf{1}$ & $\mathbf{2}$ & $\mathbf{3}$ & $\mathbf{4}$ & $\mathbf{5}$ \\
\hline RB++ & $0 / 15$ & $0 / 15$ & $0 / 15$ & $0 / 15$ & $0 / 15$ \\
\hline FilmFinder & $0 / 15$ & $0 / 15$ & $0 / 15$ & $2 / 15$ & $5 / 10$ \\
\hline Task & $\mathbf{6}$ & $\mathbf{7}$ & $\mathbf{8}$ & $\mathbf{9}$ & $\mathbf{1 0}$ \\
\hline RB++ & $0 / 15$ & $0 / 15$ & $1 / 15$ & $0 / 15$ & N/A \\
\hline FilmFinder & $4 / 7$ & $13 / 15$ & $2 / 15$ & $5 / 15$ & N/A \\
\hline
\end{tabular}

We also did paired sample t tests on three questions which were completed after each task. Each answer was a point on a 5 point scale: strongly agree (5), agree (4), neither agree nor disagree (3), disagree (2), strongly disagree (1). The following table shows the mean ratings for the first question of "It is easy to use the interface" for each task and the t-test significance levels.

Table 3. Mean ratings for the first question

\begin{tabular}{|c|c|c|c|c|c|}
\hline Task & $\mathbf{1 ~ ( . 3 3 4 )}$ & $\mathbf{2 ~ ( . 1 8 9 )}$ & $\mathbf{3}(.582)$ & $\mathbf{4}(.005)$ & $\mathbf{5}(.000)$ \\
\hline RB++ & 4.8 & 4.7 & 4.7 & 4.9 & 4.8 \\
\hline FilmFinder & 4.9 & 4.9 & 4.7 & 3.7 & 2.0 \\
\hline Task & $\mathbf{6 ~ ( . 0 0 0 )}$ & $\mathbf{7 ( . 0 0 0 )}$ & $\mathbf{8 ( . 0 0 0 )}$ & $\mathbf{9}(.000)$ & $\mathbf{1 0}(\mathbf{. 0 0 0})$ \\
\hline RB++ & 4.8 & 4.7 & 4.5 & 4.7 & 4.2 \\
\hline FilmFinder & 1.7 & 2.7 & 3.1 & 2.9 & 3.6 \\
\hline
\end{tabular}

The second question was "I feel satisfied with the results I got". The mean ratings for each task and the t-test significance levels appear in table 4.

Table 4. Mean ratings for the second question

\begin{tabular}{|c|c|c|c|c|c|}
\hline Task & $\mathbf{1 ~ ( 1 . 0 0 0 )}$ & $\mathbf{2}(\mathbf{. 1 7 3})$ & $\mathbf{3}(\mathbf{. 3 3 4})$ & $\mathbf{4}(\mathbf{. 1 0 4})$ & $\mathbf{5}(\mathbf{. 0 0 1})$ \\
\hline RB++ & 4.8 & 4.8 & 4.8 & 4.9 & 4.7 \\
\hline FilmFinder & 4.8 & 4.5 & 4.9 & 4.6 & 3.0 \\
\hline
\end{tabular}




\begin{tabular}{|c|c|c|c|c|c|}
\hline Task & $\mathbf{6 ~ ( . 0 0 0 )}$ & $\mathbf{7 ~ ( . 0 0 0 )}$ & $\mathbf{8}(\mathbf{. 0 0 0})$ & $\mathbf{9}(\mathbf{. 0 0 1})$ & $\mathbf{1 0}(\mathbf{. 0 4 8 )}$ \\
\hline $\mathrm{RB}++$ & 4.7 & 4.8 & 4.8 & 4.7 & 4.3 \\
\hline FilmFinder & 2.7 & 3.6 & 3.5 & 3.4 & 3.9 \\
\hline
\end{tabular}

The third question was "I feel confident with the results I got". The results are summarized in table 5 .

Table 5. Mean ratings for the third question

\begin{tabular}{|c|c|c|c|c|c|}
\hline Task & $\mathbf{1 ~ ( . 5 8 2 )}$ & $\mathbf{2}(\mathbf{. 6 1 0})$ & $\mathbf{3}(\mathbf{. 3 3 4})$ & $\mathbf{4}(\mathbf{. 0 0 9})$ & $\mathbf{5}(\mathbf{. 0 0 0})$ \\
\hline RB++ & 4.7 & 4.7 & 4.8 & 4.8 & 4.8 \\
\hline FilmFinder & 4.8 & 4.6 & 4.9 & 4.4 & 2.9 \\
\hline Task & $\mathbf{6 ~ ( . 0 0 0 )}$ & $\mathbf{7 ( . 0 0 1 )}$ & $\mathbf{8}(.000)$ & $\mathbf{9 ( . 0 0 1 )}$ & $\mathbf{1 0}(.110)$ \\
\hline RB++ & 4.8 & 4.7 & 4.8 & 4.8 & 4.3 \\
\hline FilmFinder & 2.5 & 3.8 & 3.4 & 3.5 & 3.9 \\
\hline
\end{tabular}

We also compared the results for the 7 overall usability questions on each interface asked after participants had done the tasks with each interface. The answer is also a five point scale from strongly agree to strongly disagree. The results and significance levels are displayed in table 6 .

Table 6. Overall ratings on usability questions

\begin{tabular}{|c|c|c|}
\hline & RB++ & Filmfinder \\
\hline Easy to use (.000) & 4.8 & 3.3 \\
\hline $\begin{array}{c}\text { Easy to navigate } \\
(.019)\end{array}$ & 4.6 & 3.9 \\
\hline Easy to browse (.001) & 4.7 & 3.5 \\
\hline $\begin{array}{c}\text { Satisfied with the } \\
\text { result (.000) }\end{array}$ & 4.7 & 3.4 \\
\hline $\begin{array}{c}\text { Confident with the } \\
\text { result (.000) }\end{array}$ & 4.6 & 3.3 \\
\hline $\begin{array}{c}\text { Fun to use (.000) } \\
\text { Help have deeper } \\
\text { understanding of the } \\
\text { collection (.001) }\end{array}$ & 4.7 & 3.7 \\
\hline
\end{tabular}

Clearly, satisfaction with the $\mathrm{RB}++$ was greater than that with the Filmfinder.

There were also open-ended questions that the participants answered after finishing both interfaces. For the first question, all of the participants considered the $\mathrm{RB}++$ interface was easier to use, especially for the complex searches with data analysis. They commented on the easy use of the visual display with the multiple categories, which made it easy to combine the search criteria and narrow down the data, and they also thought it was good to be able to manipulate the search results in multiple ways.
Thirteen out of 15 participants indicated that the $\mathrm{RB}++$ interface gave them more confidence to complete the tasks. It was easy to go back and forth and to verify the results and the informative overview panel gave the participants more confidence to finish tasks. There was one participant who thought that both interfaces gave equal confidence and there was one participant who thought that the Filmfinder interface gave more confidence since he was more familiar with the Filmfinder and he felt somewhat confused by the dynamic feature of the RB++ (such as the dynamic update of the bar when moused over), but he acknowledged the usefulness of the dynamic feature in narrowing the results in the results panel.

When asked which interface better helped them gain an understanding of the library movie collection, the RB++ interface was chosen by all the participants. Again the visual display of the multiple categories and the cross reference of these categories was considered to be useful features for them to understand the whole collection. In addition, 10 out of the 15 participants indicated that they were more likely to use the $\mathrm{RB}++$ interface if both were available, since it was easy to use and quickier to do the search. Three participants chose both interfaces, depending on the type of tasks, and two participants chose the FilmFinder because of its familiarity and aesthetic appeal.

For the question on the best thing about the $\mathrm{RB}++$, participants pointed out the visual display of the multiple categories, its cross reference ability, the dynamic matching ability of the searching boxes and the one screen display of the results as opposed to the multiple page display of results in Filmfinder. For the worst thing about the $\mathrm{RB}++$, participants indicated that it was not as aesthetically appealing as the Filmfinder and not quite as intuitive to use as the Filmfinder. Two participants specifically mentioned that the constant changing and updating of the interface made it a bit confusing. For the best thing about the Filmfinder, participants credited it with a "prettier" interface and considered it to be relatively simple to use and easy to learn. The worst things about the Filmfinder indicated by participants included the difficulty of completing type 2 tasks, lack of collection overview and multiple page display of results.

\subsection{Phase Two Results}

During the second phase we also asked participants to fill out the satisfaction questionnaires (with five point scale answer like in the phase one study) after finishing each task. Table 6 contains the mean ratings to three questions across different tasks, where relatively high ratings were given by participants :

Table 7. Overall ratings on three questions

\begin{tabular}{|c|c|c|c|c|}
\hline Task & $\mathbf{1}$ & $\mathbf{2}$ & $\mathbf{3}$ & $\mathbf{4}$ \\
\hline Easy to use & 4.5 & 4.0 & 4.2 & 3.5 \\
\hline Feel confident with results & 4.1 & 3.9 & 4.3 & 3.6 \\
\hline Feel satisfied with results & 4.1 & 4.1 & 4.4 & 3.8 \\
\hline
\end{tabular}

Participants were also required to answer a set of open questions after finishing the second phase. For the first question: "What is your overall impression of this interface for finding the statistical data?" the overall impression was positive. 
Participants used phrases such as "fairly easy to use", "very helpful in finding the information", "good for quick searching". There were also a couple of negative comments such as: "interface still came up with many results after filtering", "title of the results are not descriptive enough". Only one participant said that he did not like the interface, because of the poor categorization of information items under some categories which made him frustrated (not a function of the interface).

When answering the second question: "Was it helpful to understanding what is available at EIA?" all the participants thought the interface was helpful in that regard, which was largely attributed to the visual display of the categories, which gave them a sense of what the website covered. One participant wished that there were more categories displayed. The questionnaire also asked if the search boxes were helpful in completing the tasks. Participants gave high praise to this feature with comments such as "it's great to be taken directly to the page but not to have your results lost", "I like the way it narrows the focus and sort of guides a person to the info sought", "I didn't have to be concerned with performing a complex search that may return a null set-the results reflected my search string instantly". Two participants also commented that the feature was somewhat limited in use since relevant information may not appear in the title, or description.

For the last question where we asked for any suggestions for improving the system, participants pointed out improvements on the aesthetic issue, issues of truncated display of the description field and adding more categories.

\section{DISCUSSION}

The results strongly support that the $\mathrm{RB}++$ interface was more effective and efficient in completing type 2 tasks than the baseline interface and that users felt more confident and satisfied with the $\mathrm{RB}++$ in completing type 2 and 3 tasks. The higher effectiveness, efficiency and satisfaction gained in the RB++ resulted mainly from two aspects: the visual display of the statistical summary of the information items and the dynamic keyword searching capability in the results panel. The visualization bars helped the users understand relative proportions of items at a glance and use the posting numbers directly, which is much faster than literally counting. If we look at the BNF grammar, completion of type 2 tasks in $\mathrm{RB}++$ only required participants to explore the collection (see first option of rule A1) without submitting queries to the database and then observing and counting returned results, which are necessary steps for the baseline interface to complete the same tasks (see rule $\mathrm{B} 1$ ).

The dynamic search boxes allow users do further filtering based on certain criteria and give users feedback on the filtered results instantly and continuously, which not only encourages the users to use this function, but also improves their efficiency. Another interface feature: displaying all the results on one screen might also help improve the efficiency and satisfaction, as several users mentioned.

Several components were tightly coupled in the interface with displayed search results. The search boxes are tightly coupled with the results, which means that any input in the search boxes will invoke instant filtering on the results. The visual bars are tightly coupled with the results and as such they support two functions. One is that any operations on the visual bars such as mouse over and selection, invoke the instant filtering of the results. The other is that any update of the results also updates the summary statistics in the visualization on the bars. Coupling provides users more ways to interact with the system and make the interaction more natural and smooth (see rule A8, A9, A10), which suggests a different interaction style for finding information than traditional search interfaces which tend to require discrete, well-defined turn-taking between the user and system. Traditionally, when users get to the results page, all they can do is browse the results. If they want to refine the results, they have to go back to the search interface, type in the refined keywords, click the search, and browse the new results, which not only interrupts the normal results browsing interaction, but also loses the current result set. $\mathrm{RB}++$ encourages users to get an initial manageable result set and then refine it using one interface window without the need to go back and forth. Instead of displaying a set of static results, $\mathrm{RB}++$ offers an effective and efficient means for users to understand the results by displaying summary statistics bars which give both visual and numeric data (see rule A9), and to explore results by providing ways to dynamically and continuously filter (see rule A10). The result set can be as large as displaying the whole collection, or as small as only one item, which depends on the initial query on the collection. In the second phase study, most of the participants completed their search tasks without doing the second-time query on the initial interface. The study showed that participants could utilize the initial interface to get an initial result set by selecting relevant categories and then narrow down results and find relevant web pages by exploring the results set. Typing in keywords (or string patterns) in search boxes was found to be the most frequently used means to explore and filter result set. These features were highly appreciated by the participants as seen from their comments.

\section{RELATED WORK}

Many information access interfaces try to provide a starting point for users by presenting overviews of the collection [6]. Overviews can help users understand the whole collection and select or eliminate sources from consideration. Overviews can direct users to subcollections quickly, where they can explore the details. Usually two types of overviews are employed: category overview and graphic overview. The category approach of Yahoo is a good example for the category overview. The HiBrowse interface for viewing category labels hierarchically based on the facets is another example [12]. A more recent information access interface using the category overview by presenting faceted metadata is the Flamenco interface [19]. The last two interfaces not only present the category labels to the users but also inform the users of the number of documents under each category. However, these interfaces do not allow users to employ simple mouse moves to quickly explore the relationship between different categories (or facets). The Flamenco interface could do this as part of its browsing and searching efforts, but it requires many commitments from users such as clicking the category and waiting. The previous version of the relational browser [10] presented various categories and allowed users to explore the relations by mouse over operation, but the interface only allowed the users to mouse over the main category.

The graphic overview is another type of overview, which usually employs various information visualization techniques. Lin used the Kohenen map to visually present a topical overview 
of the collection [9]. Each block on the map represents a subcollection with similar topics which are labeled by one or two salient words extracted from the subcollection. The adjacency of blocks indicates the topic similarity between subcollections. Wise, et al. [17] developed a three dimensional interface to visually present various topics. Zhang, et al. [21] exacted the key concepts from a collection and visually presented the concepts in a spring-embedded graph. Similar concepts were clustered together and usually represented as subtopics. The graphical overview is visually appealing, but the usability of this kind of interface has yet to be explored. 3-D interfaces are more problematic than 2-D interface in terms of ease of use and learnability. It seems that textual labels of category structure are more understandable than graphical representation.

Some research has been conducted on how to present the retrieved results in certain context. Hearst [7] used clustering techniques to cluster retrieval results on the fly and presented different clusters with labeled words to the users to help them understand of the results. Chen, and Dumais [3] employed classification techniques to categorize retrieved results based on the existing category structure and displayed them in hierarchical categories. These interfaces had to cluster or categorize the retrieval results on the fly, so scaling is problematic. The $\mathrm{RB}++$ categorizes collection off line and uses a uniform category structure to present overviews of the collection and the retrieval results. Consequently, $\mathrm{RB}++$ can be scaled up easily and reduce the waiting time for the results returned. We have applied the interface to various data sets, the number of records ranging from thousands to millions ${ }^{2}$.

There also has been some work on fast location of specific information items. Sorting is a prevalent means to help users locate a specific item. However, users still need to visually go through a list of items. The Alphaslider [1] is a visual component to help users quickly locate a known string of items, but it's not very easy to use, especially for novice users. Besides, The Alphaslider can only locate the information items based on the first letter alphabetically. $\mathrm{RB}++$ provides an easy and flexible way to locate the information items by typing in string patterns and the patterns can be matched anywhere in the information items. A similar technique is actually used in some applications such as the address box of Internet Explorer browser, but the patterns are limited to matching from the very beginning.

Dynamic query was a new type of interface [14] that inspired the original relation browser work. The interface visually displays the information items and provides the visual controlling components to explore the information items by tightly coupling search and visual display of results. $\mathrm{RB}++$ is pretty much following this theme, but instead of providing a visual interface, $\mathrm{RB}++$ employs a more understandable (especially for topical overview) category structure for the information items. Moreover, the search box is a very effective and efficient component for the non categorized attributes of the items, while the visual controlling components such as sliders or check boxes can only be used for controlling categorical attributes of the items.

\footnotetext{
${ }^{2}$ Various $\mathrm{RB}++$ examples are available at http://idl.ils.unc.edu/rave/examples.html
}

Query preview [16], attribute explorer [15] and other interfaces [8], and [18] provide similar ways to explore the relationships between different facets of the classification. These interfaces worked for structured information such as database. We intend to make the interface work for the unstructured textual information. The search boxes are also provided to help users to impose constraints on other types of metadata.

\section{LIMITATIONS OF RB++ AND FUTURE WORK}

One constraint in $\mathrm{RB}++$ is the limited number of categories that can be displayed, which is affected by two factors. One factor is the screen real estate. We can partially alleviate the issue of screen real estate by utilizing a Zoomable User Interface (ZUI) to display the categories. We have experimented with integrating the Jazz toolkit [2] into the interface. Another factor is size of the memory to hold the preloaded distribution counts data, the number of which increases exponentially with increased number of displayed categories. One way to solve the issue is to only calculate part of the distribution counts data, which hopefully are most frequently used during the user's interaction with the interface. Other approaches, such as employing novel data structure were also suggested by Plaisant, et al. [11]. However, all these solutions have to sacrifice the interactivity of the interface. For example, preloading partial distribution counts data for large numbers of categories make some distributional data and visualization unavailable when users try to re-partition the information space by mouse moves.

Another constraint of $\mathrm{RB}++$ is the limited matching function of the search boxes. The interface currently matches input string patterns to the corresponding result fields on the lexical level. Matching in this level is sufficient in many cases such as matching with fields with numbers or short textual strings such as titles, but for the fields with more semantic bearing strings such as descriptions of web pages, a more sophisticated match function based on semantics might be needed.

Besides, the interface currently provides a uniform category structure for both the entire collection and the retrieved results set. This is good for its consistency. However, for the retrieved result set, a more fine-grained category structure might be better for users to understand it and conduct string searches.

In the future, we plan to work on above limitations. We are also trying to automatically generate the faceted categories displayed on the overview panel using clustering techniques [5]. Right now the categories are created manually, which certainly does not scale very well to large information collection. A big scale user study on the $\mathrm{RB}++$ interface applied specifically to different government statistical web sites will also be conducted in the near future.

Overall, the $\mathrm{RB}++$ represents an example of a highly interactive user interface that offers improved performance and satisfaction for digital library users. It can find application as the entry point for a digital library or as a way to work with large results sets returned from digital library search engines.

\section{ACKNOWLEDGMENTS}

The authors wish to thank Paul Solomon for comments on drafts of the paper and Tim Shearer for designing the underlying database structure of the interface. This work is supported by a grant from the National Science Foundation (EIA 0131824). 


\section{REFERENCES}

[1] Ahlberg, C., and Shneiderman, B. The alphaslider: a compact and rapid selector. In Proceedings of the SIGCHI conference on human factors in computing systems. Boston, Massachusetts. 1994.

[2] Bederson, B., Meyer, J., and Good, L. Jazz:An Extensible zoomable user interface graphics toolkit in java. In $A C M$ UIST2000, 171-180.

[3] Chen, H, and Dumais, S. Bringing order to the web: Automatically categorizing searching results. In Proceedings of the SIGCHI conference on human factors in computing systems. The Hague, Amsterdam. 2000

[4] Dix, A., Finlay, J. Abowd, G. Beale, R, and Finley, J. Human-Computer Interaction ( $2^{\text {nd }}$ Ed.). Prentice Hall, Hillsdale, NJ, 1998

[5] Efron, M., Marchionini, G. and Zhang, J. Implications of the recursive representation problem for automatic concept identification in on-line governmental information. ASIST SIG-CR Workshop, Long Beach, CA, 2003.

[6] Hearst, M. User interfaces and visualization. In Modern information retrieval. Ed. by Baeza-Yates, R., and RibeiroNeto, B. Chapter 10, ACM Press, New York, NY, 1999 257-324.

[7] Hearst, M. and Pedersen, P. Reexamining the cluster hypothesis: Scatter/Gather on retrieval results, Proceedings of 19th Annual International ACM/SIGIR Conference, Zurich, 1996

[8] Lanning, T., Wittenburg, K., Heinrichs, M., Fyock, C., and $\mathrm{Li}, \mathrm{G}$. Multidimensional information visualization through sliding rods. AVI'02 Palermo, Italy.

[9] Lin, X. Map displays for information retrieval. Journal of the American society for information science. 1997 48(1), 40-54.

[10] Marchionini, G., and Brunk, B. Toward a general relation browser: A GUI for information architects. Journal of Digital Information. Article No. 179, 2003-04-09 2003 $4(1)$. http://jodi.ecs.soton.ac.uk/Articles/v04/i01/Marchionini/
[11] Doan, K., Plaisant, C., Shneiderman, B., and Bruns, T. Interface and Data Architecture for Query Preview in Networked Information Systems. ACM Transactions on Information Systems, July 1999, Vol. 17, No. 3, 320-341.

[12] Pollitt A. S., Ellis G. P., and Smith M. P. HIBROWSE for Bibliographic Databases Journal of Information Science, 199420 (6), 413-426.

[13] Reisner, P., Formal Grammar and human factor design of an interactive graphics system. IEEE Trans. on Software Engineering, 7(2), 229-240, 1981

[14] Shneiderman, B., Dynamic queries for visual information seeking, IEEE Software 11, 6 (1994), 70-77.

[15] Spence, R, and Tweedie, L. The attribute explore: information synthesis via exploration. Interacting with Computers. 1998 11, 137-146.

[16] Tanin, E., Lotem, A., Haddadin, I., Shneiderman, B., Plaisant, C., and Slaughter, L. Facilitating data exploration with query previews: a study of user performance and preference. Behaviour \& information technology. 2000 19(6). 393-403.

[17] Wise, J., Thomas, J., Pennock, K., Lantrip, D., Pottier, M. and Schur, A. Visualizing the non-visual: spatial analysis and interaction with information from text documents. In Proc. of the Information visualization Symposium 95, pages 51-58. IEEE Computer Society Press, 1995

[18] Wittenburg, K., Lanning, T., Heinrichs, M., and Stanton, M. Paraell bargrams for consumer-based information exploration and choice. UIST' 01, Orlando FL.

[19] Yee, K, Swearingen, K, Li, K, and Hearst, M. Faceted metadata for image search and browsing. CHI'03, Ft. Lauderdale, FL.

[20] Zhang, J., and Marchionini, G. Relational Browser++: a Fast and Contextualized Searching and Browsing Tool. Technical Report SILS-TR-2004-01. University of North Carolina at Chapel Hill, 2004.

[21] Zhang, J., Mostafa, J., and Tripath, H. Information retrieval by semantic analysis and visualization of concept space for the D-lib magazine. D-lib online magazine, 2002 (10). 